\title{
Comments on "A New Random-Error-Correction Code"
}

\author{
Paaske, Erik
}

Published in:

I E E E Transactions on Computers

Link to article, DOI:

10.1109/TC.1979.1675329

Publication date:

1979

Document Version

Publisher's PDF, also known as Version of record

Link back to DTU Orbit

Citation (APA):

Paaske, E. (1979). Comments on "A New Random-Error-Correction Code". I E E E Transactions on Computers, C-28(3), 255-257. https://doi.org/10.1109/TC.1979.1675329

\section{General rights}

Copyright and moral rights for the publications made accessible in the public portal are retained by the authors and/or other copyright owners and it is a condition of accessing publications that users recognise and abide by the legal requirements associated with these rights.

- Users may download and print one copy of any publication from the public portal for the purpose of private study or research.

- You may not further distribute the material or use it for any profit-making activity or commercial gain

- You may freely distribute the URL identifying the publication in the public portal

If you believe that this document breaches copyright please contact us providing details, and we will remove access to the work immediately and investigate your claim. 


\section{CONCLUSION}

The error correction method proposed here makes no assumption about which bits can be in error. The correction that is performed never displaces the count more than one in the counting sequence. The decoding method is, however, essentially a code book, table look up process, and any decoder network that implements it expands rapidly with $n$.

\section{ACKNOWLEDGMENT}

The author is indebted to F. James who first discovered that some of the equations in the Kautz paper ${ }^{1}$ were in error and to other students in his EECS 538 classes who contributed ideas.

\section{Comments on "A New Random-Error-Correction Code"}

\section{ERIK PAASKE}

Abstract - This correspondence investigates the error propagation properties of six different systems using a $(12,6)$ systematic double-error-correcting convolutional encoder and a one-step majority-logic feedback decoder. For the generally accepted assumption that channel errors are much more likely to occur than hardware errors in the decoder, it is shown that the system proposed by Massey outperforms the system proposed by En in the above note. ${ }^{1}$ Further, a third system is found which, even if temporary hardware errors in the decoder are taken into account, is superior to the system proposed by En.

Index Terms - Convolutional code, error correcting code, error propagation, feedback decoder, majority logic, nonlinear feedback shift register.

\section{INTRODUCTION}

In the above paper ${ }^{1}$ En presents a "new" coding system using a $(12,6)$ systematic convolutional encoder and a one-step majoritylogic feedback decoder. En claims that the error propagation properties of his system is better than the corresponding properties of a similar system proposed by Massey [1], but unfortunately En does not define what he means by "error propagation."

All channel coding theory rests on the basic assumption that a channel error is much more likely to occur than a hardware error in the decoder. Using this basic assumption, we present appropriate and precise definitions of error propagation, of propagation length, and of propagation count in a convolutional coding system. We then show that the above claim by En cannot be true unless a definition of error propagation is used which violates this basic assumption. Finally, we show that a third system exists which is superior to the one proposed by En, even if temporary hardware errors in the decoder are taken into account, when our definitions are used.

\section{Notation AND DEFINITIONS}

Since our results are primarily based on the correspondence by Massey and Liu [2], we shall use their nomenclature and notation. The following precise definition is implicit in [2].

Manuscript received April 18, 1977.

The author is with the Institute of Circuit Theory and Telecommunication, Technical University of Denmark, DK-2800 Lyngby, Denmark.

${ }^{1}$ J. En, IEEE Trans. Comput., vol. C-26, no. 1, pp. 86-88, Jan. 1977.
Definition I: Error propagation for a feedback decoder is the event that a decoding error initiates succeeding decoding errors when all succeeding parity checks are satisfied.

We have emphasized the term decoding error in Definition I to make it clear that, with this definition, we do not take into account hardware errors in the decoder; i.e., that we make the basic assumption described above.

Definition II : Let an error propagation event start with a decoding error at time $j$ and with the syndrome register in state $s$, let all parity checks be satisfied after time $j$, and let the last decoding error from this event occur at time $i$. Then we define the state propagation length as $L_{s}=i-j$, and we define the maximum propagation length as

$$
L=\max _{s \in B} L_{s}
$$

where $B$ is the set of all reachable states [2], i.e., all states which can be reached by the syndrome register when it starts from the all-zero state.

This definition is in agreement with the one used by Robinson [4], except that we consider only states in $B$ and count $L$ in time units. A large value of $L$ indicates that, in some cases, the decoder needs a long period to reconverge to correct operation; it is noteworthy that during this period the decoder is especially vulnerable to further ordinarily correctable channel errors-it is also noteworthy that further channel errors often cause abrupt reconvergence of the decoder.

Definition III: The propagation count $\Gamma$ is

$$
\Gamma=\sum_{s \in B} \varepsilon_{s}
$$

where $\varepsilon_{s}$ is the number of decoding errors propagated from initial state $s$ in the autonomous case [2] when all parity checks are satisfied.

A large value of $\Gamma$ indicates that the amount of propagated errors may be significant for the overall error probability.

\section{Analytic Results}

There are six different $(12,6)$ systematic convolutional encoder and one-step majority logic feedback decoder systems that can correct two random errors. These are listed in Table I. System I is the system proposed by $\mathrm{En}^{1}$, system III is the one proposed by Massey [1], while Peterson and Weldon [3] use system IV as an example of a system that can produce infinite error propagation, i.e., $L=\infty$. In. Table II, we have listed the values of $L$ and $\Gamma$ for these systems. These results were obtained by the procedure outlined in [2] which applies Lyapunov's direct method to the nonlinear feedback shift register (NFSR) which is used as the feedback decoder. Definition II implies that $L=\infty$, if the NFSR is unstable and not even driven-stable [2]; system IV has $L=\infty$. Conversely, Definition II implies that $L$ is finite if the NFSR is stable or driven-stable. For systems with finite $L$ and $\Gamma$, the actual values can be determined simply by calculating $L_{s}$ and $\varepsilon_{s}$ for all $s \in B$; the calculation is facilitated by the fact that one needs only to consider the NFSR in the autonomous case. For the six NFSR's in question, $s_{5} \equiv 0$ in the autonomous case so that there are only 32 states to consider. Since only states with three or more 1 's can possibly propagate errors, only 16 states need to be checked for each system.

In general, we believe that the best system to select in practice is the one with the smallest overall bit error probability; we would like to stress that this system is not necessarily the one with the 
TABLE I

Six Different Double-Error-Correcting Coding Systems

\begin{tabular}{|c|c|c|c|c|c|c|c|c|c|}
\hline \multirow{2}{*}{$\begin{array}{l}\text { System } \\
\text { No. }\end{array}$} & \multirow{2}{*}{\multicolumn{5}{|c|}{$\begin{array}{l}\text { Sys. Convolutional } \\
\text { Encoder with Enc. } \\
\text { Polynomial } G(D) \\
g_{0} g_{1} g_{2} g_{3} g_{4} g_{5}\end{array}$}} & \multicolumn{4}{|c|}{$\begin{array}{l}\text { Feedback Decoder with } \\
\text { Input to Threshold Function }\end{array}$} \\
\hline & & & & & & $\mathrm{A}_{1}$ & $\mathrm{~A}_{2}$ & $\mathrm{~A}_{3}$ & $\mathrm{~A}_{4}$ \\
\hline I & 11 & 1 & 0 & 0 & & $s_{0}$ & $s_{1}$ & $s_{2} \oplus s_{3}$ & $s_{4} \oplus s_{5}$ \\
\hline II & 10 & 0 & 1 & 1 & 1 & $s_{0}$ & $s_{1} \oplus s_{4}$ & $\mathrm{~s}_{3}$ & $s_{5}$ \\
\hline III & 10 & 0 & 1 & 1 & 1 & $s_{0}$ & $s_{1} \oplus s_{5}$ & $s_{3}$ & $\mathrm{~s}_{4}$ \\
\hline IV & 10 & 0 & 1 & 1 & 1 & $s_{0}$ & $s_{1} \oplus s_{4}$ & $s_{2} \oplus s_{5}$ & $s_{3}$ \\
\hline $\mathrm{v}$ & 10 & 0 & 1 & 1 & 1 & $s_{0}$ & $s_{1} \oplus s_{2} \oplus s_{5}$ & & $\mathrm{~s}_{4}$ \\
\hline VI & 11 & 0 & 1 & 1 & 1 & $s_{0}$ & $\mathrm{~s}_{1}$ & $\mathrm{~s}_{2} \oplus \mathrm{s}_{5}$ & $\dot{s}_{3}$ \\
\hline
\end{tabular}

TABLE III

Simulation Results for the Coding Systems in Table I: PropaGATION FACTOR $R_{f}$ BASED ON $\mathbf{4 0} 000$ INFORMATION BITS TRANSMITTED Over the BSC WITH Crossover Probability $p$

\begin{tabular}{|l|c|c|c|c|c|}
\hline \hline \multirow{2}{*}{$\begin{array}{c}\text { SYstem } \\
\text { No. }\end{array}$} & \multicolumn{5}{|c|}{ Propagation Factor $\mathrm{R}_{\mathrm{f}}$} \\
\cline { 2 - 6 } & $\mathrm{p}=0.10$ & $\mathrm{p}=0.08$ & $\mathrm{p}=0.06$ & $\mathrm{p}=0.04$ & $\mathrm{p}=0.02$ \\
\hline I & 2.1 & 2.3 & 2.2 & 2.1 & 2.1 \\
II & 2.0 & 2.0 & 2.0 & 1.9 & 1.8 \\
III & 1.9 & 2.0 & 2.1 & 1.9 & 1.7 \\
IV & 2.3 & 2.4 & 2.7 & 3.2 & 2.8 \\
V & 2.0 & 2.1 & 2.3 & 2.2 & 1.6 \\
VI & 2.4 & 2.5 & 2.6 & 2.4 & 2.5 \\
\hline
\end{tabular}

smallest amount of error propagation. However, calculation of the overall bit error probability is very difficult and depends on the particular channel. Therefore, if the choice among different systems has to be based only on simple analytic calculations, we believe that the best system to select is the one with the smallest values of $L$ and $\Gamma$. Thus from Table II it follows that system II could generally be expected to be the best; it also follows that the system proposed by Massey [1], system III, could generally be expected to outperform the system proposed by En, ${ }^{1}$, system I.

Finally, we consider the effect of a temporary hardware error in the decoder. Under such circumstances, the syndrome register can reach a state $s \notin B$. If $L_{s}=\infty$ for this state, then an infinite number of succeeding decoding errors results if all further parity checks are satisfied; but this can happen only when the NFSR is not stable. Thus the interesting case is, of course, when the NFSR is not stable but is driven-stable. Hence, for system III, a temporary hardware error in the decoder can produce a situation which looks like "infinite error propagation," but this cannot happen for systems I, II, V, and VI. This phenomenon apparently led En to claim that, as regards error propagation, system I outperforms system III. But, as can be seen from Table II, En's claim is only true if the basic assumption stated in the introduction is abandoned. Since system II has a stable NFSR, it can also be seen from Table II that, even if temporary hardware errors were taken into account, this system could still be expected to outperform the other systems.

\section{Simulation Results}

To test the appropriateness of using $L$ and $\Gamma$ to select the system with the smallest amount of error propagation, a simulation was performed using the method given by Sullivan [5]. This method uses, for comparison purposes, a "genie-aided" feedback decoder which feeds back the true channel error $e_{u}^{i}$ instead of the estimated
TABLE II

Analytic Results of Error Propagation Properties FOR THE CODING SYSTEMS IN TABLE I

\begin{tabular}{|l|l|c|c|}
\hline $\begin{array}{c}\text { System } \\
\text { No. }\end{array}$ & $\begin{array}{c}\text { Results of Applying Lyapunov's } \\
\text { Method on Feedback Decoder }\end{array}$ & $\begin{array}{c}\text { Propagation } \\
\text { Length L }\end{array}$ & $\begin{array}{c}\text { Propagation } \\
\text { Count } \Gamma\end{array}$ \\
\hline I & $\bar{A}_{11}=\Phi \mid$ Stable & 5 & 16 \\
II & $\bar{A}_{8}=\Phi \mid$ Stable & 3 & 4 \\
III & $\mathrm{S}_{9}=\Phi, \bar{A}_{8}=23 \mid$ Driven Stable & 3 & 9 \\
IV & $\mathrm{S}_{10}=\Phi, \overline{\mathrm{A}}_{9}=13,14,15,26,27,30 \mid$ & & \\
V & $\bar{A}_{11}=\Phi \mid$ Stable $\quad$ Unstable & 5 & 17 \\
VI & $\bar{A}_{11}=\Phi \mid$ Stable & 5 & 17 \\
\hline
\end{tabular}

TABLE IV

Simulation Results for the Coding Systems in TABLe I: Number OF INFORMATION DeCODING ERRORS AMONG 40000 INFORMATION Bits

TRANSMITTED OVER THE BSC WITH CROSSOVER PROBABILITY $p$
\begin{tabular}{|l|c|c|c|c|c|}
\hline \hline \multirow{2}{*}{$\begin{array}{c}\text { System } \\
\text { No. }\end{array}$} & \multicolumn{6}{|c|}{$\begin{array}{c}\text { No. of Information Decoding Errors } \\
\text { with Feedback Decoding }\end{array}$} \\
\cline { 2 - 7 } & $\mathrm{p}=0.10$ & $\mathrm{p}=0.08$ & $\mathrm{p}=0.06$ & $\mathrm{p}=0.04$ & $\mathrm{p}=0.02$ \\
\hline I & 3627 & 2279 & 1170 & 400 & 35 \\
II & 3220 & 1910 & 999 & 350 & 32 \\
III & 3208 & 1961 & 1017 & 344 & 32 \\
IV & 3754 & 2328 & 1317 & 526 & 68 \\
V & 3386 & 2150 & 1131 & 374 & 33 \\
VI & 3953 & 2469 & 1284 & 442 & 58 \\
\hline
\end{tabular}

value $e_{u}^{i \Delta}$. In other words, erroneous feedback never occurs and error propagation is thus impossible. We define the propagation factor $R_{f}$ to be the ratio between the number of information decoding errors made by a system with feedback decoding over that of a "genie-aided" feedback decoder with the same decoding function. We used a binary symmetric channel with crossover probability $p$. For each value of $p, 40000$ information bits were transmitted for each system.

Table III shows the values of the propagation factor $R_{f}$. Except for system VI for which the values of $R_{f}$ are slightly larger than expected, we observe a good agreement with the selection criteria outlined above. In Table IV we have also listed the total number of decoding errors made by each system. Clearly systems II and III outperform the other systems in the sense of minimizing the overall error probability for the channels considered.

\section{Concluding Remarks}

The majority logic feedback decoder was originally developed under the assumption that channel errors are much more likely to occur than temporary hardware errors in the decoder; the appropriateness of a feedback decoder is questionable if this assumption is violated. Thus we conclude that, if the systems are used in an appropriate way at all, the system proposed by Massey outperforms the system proposed by En. However, in general, we would recommend system II over either since system II minimizes overall error probability and, furthermore, cannot produce a situation which looks like "infinite error propagation" even if temporary hardware errors in the decoder occur.

As a final remark, we mention that determination of the propagation length $L$ based on application of Lyapunov's method to the NFSR of the decoder is to our knowledge the best way to analyze error propagation for codes with a relatively small register, i.e., codes with rates $R \geq \frac{1}{2}$. For codes with $R<\frac{1}{2}$ where the number of 
syndrome states becomes large, it often pays to consider instead the postdecoded bits $e_{u-j}^{i \Delta \Delta}=e_{u-j}^{i \Delta} \oplus e_{u-j}^{i \Delta}, j=1,2, \cdots, m$, as was done by Sullivan [5] in investigating the error propagation properties of uniform codes. We would like to point out, however, that, after a slight modification, Lyapunov's method, can be applied to the postdecoded bits to determine $L$.

\section{ACKNOWLEDGMENT}

The author wishes to thank Prof. J. L. Massey, M.I.T., Cambridge, MA, for his assistance to clarify the final manuscript.

\section{REFERENCES}

[1] J. L. Massey, Threshold Decoding. Cambridge, MA: MIT Press, 1963.

[2] J. L. Massey and R. W. Liu, "Application of Lyapunov's direct method to the error-propagation effect in convolutional codes," IEEE Trans. Inform. Theory, vol. IT-10, pp. 248-250, July 1964.

[3] W. W. Peterson and E. J. Weldon, Jr., Error Correcting Codes. Cambridge, MA: MIT Press, 1972.

[4] J. P. Robinson, "Error propagation and definite decoding of convolutional codes," IEEE Trans. Inform. Theory, vol. IT-14, pp. 121-128, Jan. 1968.

[5] D. D. Sullivan, "Error propagation properties of uniform codes," IEEE Trans. Inform. Theory, vol. IT-15, pp. 152-161, Jan. 1969.

\section{Author's Reply ${ }^{2}$}

\section{JOHN EN}

\begin{abstract}
Without proof Paaske ${ }^{3}$ states that "... En" cannot be true." Paaske's statement is based on his qualitative assumption that channel error is "much more likely" to occur than a hardware error in the decoder. The author will show that En's statements are still true under Paaske's assumption. Previous correspondence was cited by Paaske and claimed to be "the best way ..." to judge a code based "on the particular channel" and $\mathbf{4 0 0 0 0}$ test pattern bits. Hence, he claims that the system II and the Massey code outperform the En code. However, the En code can outperform both system II and the Massey code under other channel characteristies and other sets of test bits. Therefore, the method quoted by Paaske is inconclusive at best. Massey's Threshold Decoding is a significant contribution. Regardless of his original assumption, his particular decoder does propagate infinite errors. However, this should not be considered as criticizing Massey's work. The En code has been proved empirically to be free from catastrophic errors under any error sources. Since Paaske did not prove anything to the contrary, all of En's original claims stand as before.
\end{abstract}

Index Terms - Catastrophic error propagation, convolution code, feedback decoder, infinite error propagation, majority logic decoder.

In the accompanying correspondence, ${ }^{3}$ there are several points made which are subject to discussion. Paaske makes the statements:

“... basic assumption ... channel error is much more likely to occur than a hardware error ..." “... above claim by En cannot be true unless a definition of error propagation is used which violates this basic assumption." (Introduction, paragraph 2) ${ }^{3}$

There are two problems with that statement. First, "much more

\footnotetext{
${ }^{2}$ Manuscript received April 18, 1978.

The author is with Advanced Technology, Cubic Corporation, P. O. Box 80787, San Diego, CA 92138.

${ }^{3}$ E. Paaske, "Comments on 'A New Random Error Correction Code'," this issue, pp. 255-257.
}

likely" is not a sufficient premise to permit the judgment "... cannot be true...." In order to make such a statement Paaske would have to state that there are absolutely no other error sources existent except channel errors. Since Paaske cannot realistically assume such a premise, Paaske's statement is unfounded. A decoder need only to have a catastrophic failure once, and even the poorest decoder without a catastrophic failure mode can be judged as a better code.

Second, as will be discussed, En's claims are not based on Paaske's basic assumptions; they are based on En's basic assumptions.

Paaske also states:

“... calculation of the overall bit error probability is very difficult, and depends on the particular channel.... choice... based only on simple analytic calculations, ..."

and:

“... best system ... smallest overall bit error probability ...”.

These statements indicate contradiction. His choice is based only on a particular channel characteristic and a very limited number of test pattern bits (40000). Paaske's judgments can be easily reversed under other circumstances. The En code can outperform both system II and the Massey code, even under Paaske's criteria. The method cited by Paaske which he considered to be "the best" is trivial and inconclusive at best. Chien [1], Gallager [2], En, and other mathematicians agree that a better code is a code that suits the noise environment best. Even a simple repeat of message may be better than any error-correcting code for low-noise wire communications. Chien and En both agree that a good defense should be prepared before any presentation. It is apparent that Paaske's "best way" needs strong proof in order to make that claim.

The most controversial statement:

"En's claim is only true if the basic assumption stated in the introduction is abandoned."

is simply a reiteration of the statements made in Paaske's introduction. Again, it must be remembered that the basic assumption to which reference is made is Paaske's basic assumption, not En's, and contains the evasive statement "... much more likely ...," which is hardly justification for such a strong statement.

It is clear that the Massey code fails catastrophically (produces an infinite string of errors) where the En code does not. It is also clear that En in the original paper made claims based on the following:

1) All error sources, including hardware errors, are considered.

2) Error propagation is considered to be infinite error propagation, as defined in Peterson and Weldon [3].

3) Finite error propagation is not discussed because of uncertain noise environment. In fact, Peterson and Weldon also stated, "In this book finite error propagation is not considered" [3, p. 400, footnote].

4) Comprehensive testing of a code is much more reliable than simplified analytical calculations.

5) A "one of the best" code does not propagate errors, and absolutely corrects two bits in twelve consecutive bits.

Given that these are En's basic definitions, and that they are the definitions upon which a choice is made, En's code outperforms Massey's. Paaske has not addressed this point directly; he has redefined his bases (particularly 1), 2), 3), and 4) above) so as to not consider all sources of error, and he then has made unqualified statements as to the validity of En's claims.

All of the systems which Paaske proposed are available from 